\title{
Las revoluciones de Thomas Kuhn, una mirada discontinuista
}

\author{
Pablo Melogno
}

Universidad de la República

Facultad de Información y Comunicación

Montevideo, Uruguay

pablo.melogno@fic.edu.uy

Received: 05.06.2015; Revised: 26.10.2015; Accepted: 29.10.2015

DOI: http://dx.doi.org/10.1590/0100-6045.2015.V38N3.PM

Resumen: Se discuten algunas divergencias entre La revolución copernicana (1957) y La estructura de las revoluciones cientificas (1962), de Thomas Kuhn. Se muestra que presentan una consideración diferente de los factores extracientíficos, que tienen en la primera un peso explicativo mayor. Se propone que en 1957 Kuhn maneja una historiografía casuística, centrada en la revolución copernicana, y en 1962 una historiografía estructural, centrada en los rasgos invariantes de las revoluciones científicas. Se concluye que las diferencias entre las dos obras son lo suficientemente profundas como para no considerarlas expresión de una misma visión de la ciencia.

Palabras clave: filosofía de la ciencia, historiografía de la ciencia, revolución copernicana, giro historicista, revoluciones científicas.

\section{Thomas Kuhn's Revolutions, a discontinuist view}

\begin{abstract}
The paper discusses certain discrepancies between The Copernican Revolution (1957) and The Structure of Scientific Revolutions (1962) by Thomas Kuhn. It shows that they present a different consideration of extra-scientific factors, that in the former have a higher explanatory weight. It states that in 1957 Kuhn applies a casuistical historiography, focused on the Copernican revolution, and in 1962 a structural historiography, focused on the invariable features of scientific revolutions. The paper concludes that the differences between these two works are significant enough so as not to be considered an expression of the same view of science.
\end{abstract}

Manuscrito - Rev. Int. Fil., Campinas, v. 38, n.3, pp. 25-57, set.-dez. 2015. 
Keywords: philosophy of science, historiography of science, copernican revolution, historical turn, scientific revolutions.

\section{Introducción}

Thomas Kuhn es universalmente conocido por La estructura de las revoluciones cientificas -en delante Erc- de 1962, y suele presentárselo como uno de los responsables de reorientar las relaciones entre filosofía e historia de la ciencia en la segunda mitad del siglo XX. Es frecuente leer y escuchar que Kuhn abordó en su principal obra una amplia serie de problemas filosóficos, bajo una perspectiva divergente con la filosofía de la ciencia tradicional. Esta faceta de Kuhn como historiador de la ciencia con pretensiones filosóficas es por demás conocida. No obstante, sus dos trabajos mayores de historia de la ciencia, en general no han recibido la misma atención que otros sectores de su obra; nos referimos a La revolución copernicana -en delante $R_{c}-$, de 1957, y La teoría del cuerpo negro y la discontinuidad cuántica 1894-1912, de 1978. Ambos libros ocupan un rol secundario en la literatura sobre Kuhn, por más que él mismo insinuó ciertas relaciones entre Erc y sus textos históricos ${ }^{1}$.

Dentro de la escasa producción sobre la obra temprana de $\mathrm{Kuhn}^{2}$, parece haber dos tendencias relativamente definidas. La primera, tiende a considerar a las obras previas a Erc-fundamentalmente las conferencias Lowell de 1951, Rc, y algunos artículos breves-, como expresión de una misma línea de pensamiento, que anticipa desde principios de la década de los 50's y sin rupturas, la imagen de la ciencia que Kuhn defenderá en 1962. El obituario de John Heilbron publicado en Isis (1998), más los trabajos de Noel Swerdlow (2004) y James Marcum (2005), son expresión de esta forma de comprender el asunto, a la que denominaremos perspectiva continuista.

El mismo Kuhn fomentó activamente esta visión de su desarrollo intelectual. En el prefacio a La tensión esencial (1977), rememoró su trabajo como historiador de la ciencia en Harvard en las décadas de 1940 y 1950, época de

\footnotetext{
${ }^{1}$ A este respecto, Ian Hacking señala que Kuhn entendía al estudio sobre el cuerpo negro como un caso de las tesis filosóficas de Erc (Hacking en Kuhn 1962, p. 10).

2 Poca en comparación a la inmanejable cantidad de producción sobre Erc y su trabajo posterior.
} 
preparación de su estudio sobre la revolución copernicana, ubicando en dicho período el origen de algunas de las ideas que luego tomarían forma en Erc ${ }^{3}$. Situándose en 1947, se refiere al cambio en su manera de leer los textos antiguos, que le permitió dar sentido a los escritos de Aristóteles. Para el testimonio que en 1977 Kuhn ofrece de este descubrimiento hermenéutico, resulta central la noción de cambio de significado, de data muy posterior en su pensamiento "al leer las obras de un pensador importante, busca primero las absurdidades aparentes del texto, y luego pregúntate cómo es que pudo haberlas escrito una persona inteligente. Cuando tengas la respuesta, prosigo, cuando esos pasajes hayan adquirido sentido, encontrarás que los pasajes primordiales, esos que ya creías haber entendido, han cambiado de significado." (Kuhn, 1977, pp. 12$13 / 12)^{4}$

La continuidad entre Erc y su obra previa, es prolongada por Kuhn situando la primera exposición de sus ideas acerca de las revoluciones científicas en las Conferencias Lowell de $1951^{5}$, y estableciendo una línea de desarrollo que prosigue en $\mathrm{R} c$, y "La estructura histórica del descubrimiento científico" publicado en el mismo 1962 (Kuhn, 1977, p. 17/17). Finalmente, a lo largo de Erc, Kuhn no ahorró referencias a su estudio de 1957, presentándolo como una suerte de caso ilustrativo de nociones como paradigma, ciencia normal y revolución científica (Kuhn, 1962, p. 97/X, p. 198/69, p. 218/83, p. 264/117, p. $310 / 150)$.

Entendemos que a través de estas y otras referencias, el mismo Kuhn generó una imagen de continuidad entre su obra temprana sus trabajos de madurez, y particularmente entre sus dos primeros libros. Kuhn introdujo así una narrativa de su trabajo temprano que reducía sus escritos de la década del $50 \mathrm{a}$ pasos preparatorios de las celebradas ideas de Erc. Esto supuso entre otras cosas reconstruir su proceso intelectual de los años 50's apoyándose en una

\footnotetext{
3 Agradecemos especialmente a dos evaluadores anónimos de Manuscrito, por esta y otras sugerencias que contribuyeron sustantivamente a mejorar el texto original.

${ }^{4}$ En todas las citas de Kuhn, el primer número corresponde a la página de la edición en español consignada en la bibliografía, y el segundo a la página correspondiente de la edición en inglés. Para La tensión esencial, la primera edición de 1977, para $\mathrm{R} c$, la decimoctava reimpresión de 1995, y para Erc la segunda edición de 1970).
}

${ }^{5}$ Aún inéditas. Puede hallarse revisiones de interés en Marcum (2005) y Mayoral (2013).

Manuscrito - Rev. Int. Fil., Campinas, v. 38, n.3, pp. 25-57, set.-dez. 2015. 
terminología que sólo desarrollaría años después; así en 1977 se refiere a su encuentro con Aristóteles a finales de los 40's en términos de cambios en el lenguaje y la naturaleza, expresiones que sólo tomaran forma en sus escritos de comienzos de la década de 1960: "lo que pareció revelarme mi lectura de Aristóteles fue una especie de cambio generalizado de la forma en que los hombres concebían la naturaleza y le aplicaban un lenguaje, una concepción que no podría describirse propiamente como constituida por adiciones al conocimiento o por la mera corrección de los errores uno por uno." (Kuhn, 1977, p. 13/xiii)

La imagen así forjada ha sido desde entonces reproducida sin controversia por la mayor parte de la crítica, impidiendo detectar las discontinuidades de fondo que existen entre Erc y el trabajo del joven Kuhn historiador de la ciencia. El escrito de Heilbron (1998) ${ }^{6}$ asume de modo explícito esta visión que el mismo Kuhn insinuó acerca de su obra, según la cual las ideas que dieron notoriedad al pensamiento kuhniano a partir de 1962 ya se encuentran insinuadas en los trabajos de la década del 50, de modo que las tesis de Erc no son sino la consumación de un proyecto historiográfico que venía forjándose casi ladrillo a ladrillo- en los quince años anteriores.

Una segunda postura tiende a considerar a $R c$ y Erc como dos obras que presentan diferencias historiográficas de importancia, indicadores de una reorientación del pensamiento de Kuhn a finales de la década de los 50's. El comentario fermental de Elia Bravo (1979) es una referencia temprana de esta tendencia en la literatura de habla hispana, mientras que trabajo de Robert $\mathrm{S}$. Westman (1994), es seguramente su mejor expresión, aunque quizás no la más enfática. Otro intento reciente de explorar las tensiones en el desarrollo del pensamiento de Kuhn, es del de J. C. Pinto de Oliveira (2012), quien -partiendo de un período y una serie de problemas bien diferente a lo que nos interesa aquíseñaló divergencias entre la explicación que Kuhn propone para el origen de la 'nueva historiografía de la ciencia' en Erc y otros trabajos de principios de los 60's, y la visión que ofrece en el artículo "Historia de la ciencia" de 1968, incluido en La tensión esencial.

\footnotetext{
${ }^{6}$ Cabe recordar que Heilbron fue primero estudiante y luego colaborador de Kuhn, lo que tuvo como resultado el trabajo en co-autoría de 1969 (Heilbron; Kuhn 1969).

${ }^{7}$ Según Pinto de Oliveira, el primer grupo de artículos ofrece una explicación endógena del surgimiento de la nueva historiografía de la ciencia, basada en que la historiografía
} 
La postura que defendemos aquí se orienta en esta segunda línea, que llamaremos perspectiva discontinuista del pensamiento de Kuhn. La posición discontinuista implica conceder que, si bien el trabajo de Kuhn de 1957 contiene ciertas inquietudes y problemas que anticipan los desarrollos de su obra madura, éstos conviven con formulaciones y presupuestos historiográficos incompatibles con las tesis principales de Erc. En otros trabajos hemos afirmado que los marcos filosóficos que Kuhn maneja en ambas obras son incompatibles, sobre todo en lo que hace a la noción de verdad, la base empírica de la ciencia, y la función de los esquemas conceptuales en la construcción del conocimiento científico (Melogno; Pelegrin, 2015/ Melogno; Ache, 2015). En ambos textos hemos señalado algunas relaciones de proximidad entre la concepción historiográfica de Rc y la 'historiografía tradicional de la ciencia', objeto de cuestionamiento en el célebre prefacio de Erc, y cuyos presupuestos han sido analizados con detalle por Hoyningen-Huene (1993).

Hemos defendido que la manera en que en $R c$ se consideran tópicos como la relación teoría-observación, la posibilidad de un progreso científico acumulativo, y el valor de verdad de las teorías científicas, emparentan en algunos casos muy estrechamente al planteo de Rc con la antigua historiografía que luego Kuhn contribuirá a derrumbar. Aquí nos centraremos en las discrepancias de supuestos historiográficos que entendemos existen entre las dos primeras obras de Kuhn, dejando constancia de que se trata de un capítulo acotado en una serie de diferencias de más amplio alcance.

El contexto en el que Kuhn elabora su estudio sobre la revolución copernicana es relevante para comprender el alcance del libro y sus objetivos, y también para determinar por qué su contenido revela tan poco del Kubn filósofo, que hará fortuna años más tarde ${ }^{8}$. Según señala Swerdlow (2004), se trata de la primera publicación mayor de Kuhn sobre historia de la ciencia, previo a la cual solo registraba unos pocos trabajos sobre historia de la química en el siglo XVII y sobre el ciclo de Carnot. El libro fue escrito mientras Kuhn tomaba una beca

tradicional resultaba estéril frente a los descubrimientos que resultan incompatibles con las asunciones conceptuales y las expectativas de la ciencia normal. Mientras que en el escrito de 1968, Kuhn propone una explicación exógena del proceso, basa en la influencia de la historia de la filosofía, la obra de Pierre Duhem y la historia externa en el desarrollo de la nueva historiografía.

${ }^{8}$ Algunos de estos pormenores se revisan en Guillaumin (2012).

Manuscrito - Rev. Int. Fil., Campinas, v. 38, n.3, pp. 25-57, set.-dez. 2015. 
Guggenheim, durante 1954-1955, estando casi completo para 1956. El análisis de Swerdlow (2004, pp. 69-70) pone de manifiesto que la concepción general de la ciencia que Kuhn sostiene en $\mathrm{R} c$ está directamente influida por el pensamiento de James B. Conant, rector de la Universidad de Harvard y mentor de Kuhn. En On Understanding Science de 1947, Conant había expuesto el ideario educativo que inspiraba los cursos de historia de la ciencia en Harvard, a los que Kuhn fue convocado como docente y que tuvieron como resultado su libro sobre la revolución copernicana. En éste, Kuhn incorpora tanto el vocabulario como la imagen general de la ciencia que Conant defendía, lo que permite explicar buena parte de las diferencias entre $R c$ y su obra posterior.

Para identificar estas diferencias y evaluar su tenor, nos ocuparemos de algunas relaciones entre Erc y el estudio sobre la revolución copernicana de 1957, que a nuestro entender muestran que ambas obras parten de presupuestos historiográficos divergentes, lo que impide considerarlas como expresión de una línea de investigación común. Desde este punto de vista, pueden identificarse una serie de premisas historiográficas incompatibles entre la imagen específica de la revolución copernicana que Kuhn presenta en 1957 y la imagen general de las revoluciones científicas -incluida la copernicana- que ofrece en 1962. La comparación también arroja elementos para pensar que buena parte de las tesis y conceptos que hicieron célebre a Kuhn -ciencia normal, paradigma, inconmensurabildad, cambio de mundo, entre otros-, estaban aún lejos de asomar en la primera mitad de la década de los 50's.

En la sección segunda comenzamos revisando algunos supuestos historiográficos compartidos en las dos obras de Kuhn, que han dado pie a las interpretaciones continuistas de su pensamiento. En la sección tercera mostramos la primera de estas divergencias, relacionada con la muy diferente consideración de los factores sociales y extracientíficos en $\mathrm{R} c$ y Erc, debido a que en esta última, la tesis del aislamiento de las comunidades científicas deja escaso lugar para la consideración de los factores externos a la ciencia. La sección cuatro aborda un ejemplo concreto de esto, analizando el papel que desempeñan en la revolución copernicana la filosofía neoplatónica, los frentes de oposición no científica al copernicanismo, y los astrónomos ptolemaicos del siglo XVII. El análisis busca mostrar que estos factores tienen en $R c$ más peso explicativo que en Erc, a causa de las diferencias de dominio de análisis que hay en ambas obras.

La sección cinco muestra que en $\mathrm{R} c$ el cambio revolucionario registra un carácter global, afectando desde la ciencia hasta la sociedad en su conjunto, 
mientras que en Erc la consideración el cambio paradigmático se agota casi totalmente en el ámbito de las comunidades científicas. En la sección sexta revisamos el uso que Kuhn hace en ambas obras de la psicología de la Gestalt, en relación a la noción de revolución científica y a la idea de cambio de mundo; defendemos que la incorporación de la Gestalt arroja resultados muy diferentes en cada caso, ya que las implicancias filosóficas que Kuhn extrae en 1962 son inviables bajo el marco conceptual de 1957. En la sección siete revisamos el tipo de proyecto historiográfico que Kuhn emprende en cada obra, proponiendo que Rc responde a una historiografía casuística, centrada en el caso de la revolución copernicana, mientras que Erc responde a una historiografía estructural, centrada en la identificación de rasgos comunes a las diferentes revoluciones científicas. Finalmente, concluimos que si bien las dos primeras obras de Kuhn presentan algunos puntos de contacto, las diferencias entre ambas son lo suficientemente profundas como para no considerarlas como expresión de una misma visión de la ciencia, sino que más bien el período de 1957 a 1962 debe ser entendido como la transición entre dos modelos historiográficos en ciertos aspectos inconciliables.

\section{La pluralidad estructural de las revoluciones científicas}

Un aspecto importante para comprender la evolución del pensamiento de Kuhn desde 1957 a 1962 consiste en precisar de qué tipo de unidad de análisis se ocupa $R c$, y como esto se contrapone a la base historiográfica de Erc. Una revisión inicial de ambas obras puede dar la impresión de que se asientan sobre una base común de herramientas historiográficas, no obstante un análisis más detenido muestra que las discrepancias superan a las convergencias.

Comenzando con $\mathrm{R} c$, Kuhn presenta los propósitos del libro en una perspectiva que denota la influencia de Alexandre Koyré ${ }^{9}$, al señalar que su principal objetivo es mostrar la pluralidad estructural de la revolución copernicana, es decir la diversidad de disciplinas y niveles que están involucrados en el

\footnotetext{
9 Sobre la influencia de Koyrè en Kuhn, son de relieve los trabajos de Pardo (2001), y Estrada (2005). La influencia de Koyré y Herbert Butterfield también ha sido analizada en Cohen (1994, pp. 124 y ss.), Swerdlow (2004, pp. 74-75) y Marcum (2005, pp. 10 y ss.).
}

Manuscrito - Rev. Int. Fil., Campinas, v. 38, n.3, pp. 25-57, set.-dez. 2015. 
surgimiento del copernicanismo. Originalmente se trata de un cambio dado en el reducido dominio de la astronomía matemática, pero que termina provocando modificaciones de fondo en varias disciplinas científicas, y en la forma de ver el mundo en la cultura occidental.

En este primer aspecto puede verse un antecedente de la noción de paradigma, en cuanto Kuhn -siguiendo a Koyré- comienza a visualizar que el cambio científico no supone solamente la sustitución de las hipótesis contenidas en una teoría, sino que conlleva modificaciones en los valores, los supuestos metafísicos y la cosmovisión de los científicos; de manera que "A pesar de que De Revolutionibus consiste básicamente en un conjunto de fórmulas matemáticas, tablas y diagramas, sólo podía ser asimilado plenamente por hombres capaces de crear una nueva física, una nueva concepción del espacio y una nueva idea de la relación del hombre con Dios." (1957, p. 10/vii). Estos términos parecen reafirmarse en Erc, en referencia al carácter global del cambio paradigmático: "Ver oxígeno en lugar de aire desflogistizado, ver un condensador en lugar de la botella de Leyden o un péndulo en lugar de una caída obstaculizada, era sólo una parte de un cambio integral en la visión científica de una gran cantidad de fenómenos relacionados... Los paradigmas determinan al mismo tiempo grandes áreas de la experiencia." (Kuhn, 1962, p. 281/129)

Bajo la concepción historiográfica que Kuhn suscribía en 1957, la pluralidad de la revolución copernicana obliga a apelar a la historia de las ideas en complemento con la historia de la ciencia, y a ponderar la influencia de los factores extracientíficos en los procesos de elección de un marco teórico: "Los astrónomos estaban preparados en otros campos del conocimiento científico, a la vez que se hallaban inmersos en el seno de diversos sistemas filosóficos y religiosos. Un buen número de sus creencias extra-astronómicas desempeñaron un papel fundamental... modelando la revolución copernicana." (1957, p. 10/vii). El mismo argumento se repite en Erc, cuando afirma "Los científicos individuales abrazan un nuevo paradigma por todo tipo de razones y normalmente por varias a la vez. Algunas de estas razones caen por fuera de lo que aparentemente es la esfera plenamente científica, como por ejemplo el culto al Sol que contribuyó a hacer de Kepler un copernicano.” (1962, p. 315/142) ${ }^{10}$

${ }^{10}$ No sólo este argumento, sino también el ejemplo del culto al Sol se repite en las dos obras, con mayor detalle histórico en Rc (1957, pp. 179-182/130-132).

Manuscrito - Rev. Int. Fil., Campinas, v. 38, n.3, pp. 25-57, set.-dez. 2015. 
La apelación a la historia de las ideas como complemento de la historia de la ciencia, y la alusión al estudio de las creencias extra-astronómicas de los astrónomos dan cuenta de la necesidad que tenía Kuhn ya en la década de los 50's, de ampliar la unidad de análisis de la historiografía tradicional, e ir más allá de la historia de la ciencia entendida como historia de las teorías científicas y de los métodos utilizados para su construcción ${ }^{11}$.

Hasta aquí, el marco historiográfico de $R c$ muestra una continuidad con Erc, dada por la necesidad de ampliar la unidad de análisis de la historia de la ciencia, y por la consideración del carácter global del cambio revolucionario, ambas características comunes a las dos obras. Esto llevó a J. Marcum a postular una continuidad global entre ambos libros, que como señalamos, pretende remontarse hasta principios de la década de 1950:

El desarrollo de la nueva imagen de la naturaleza de la ciencia de Kuhn comenzó con las conferencias Lowell de 1951. En estas conferencias, Kuhn esbozó una concepción dinámica de la ciencia en contraste a la concepción estática de la filosofía de la ciencia tradicional. La dialéctica básica que se encuentra en sus escritos posteriores estaba presente en estas conferencias. En La revolución copernicana, continuó refinando su aproximación historiográfica a la ciencia ${ }^{12}$. En tres ensayos que siguen a La revolución copernicana, Kuhn retornó a los problemas filosóficos que animaron su interés original en la historia de la ciencia, y continuó explorando estos problemas en términos y conceptos que presagiaban a La estructura. (Marcum, 2005, p. 30) ${ }^{13}$

Previamente, R. Heilbron había expuesto también esta visión, llegando al extremo de reconstruir la narrativa histórica de $\mathrm{R} c$ con el lenguaje de Erc. Refiriéndose a la primera afirma: "los problemas con el sistema ptolemaico, en

11 "Los conceptos científicos son ideas, y como tales forman parte de la historia intelectual... estoy plenamente convencido de que las técnicas desarrolladas por los historiadores de las ideas pueden proporcionar un tipo de comprensión de los problemas que no podrá llegarle a la ciencia por ningún otro camino." (Kuhn 1957, p. 11/viii). Una valoración interesante de este particular se puede hallar en Westman (1994, p. 86-87).

${ }^{12}$ Destacado nuestro.

${ }^{13}$ Todas las traducciones nuestras en los casos en que la fuente se consigna en inglés.

Manuscrito - Rev. Int. Fil., Campinas, v. 38, n.3, pp. 25-57, set.-dez. 2015. 
su aparato técnico y en su ajuste con la observación, se habían acumulado por un milenio antes de que el occidente latino recuperara los escritos de los antiguos astrónomos griegos. Copérnico fue el primer europeo lo suficientemente afectado por estas 'anomalías' (para usar el término de La estructura)..." (Heilbron 1998, p. 508) El efecto de este tipo de hermenéutica nos parece tan claro como sesgado: si se reconstruye el trabajo de Kuhn de 1957 en términos de anomalías, paradigmas, conversiones, cambios de mundo e inconmensurabildiad, se bloquea la posibilidad -por demás pertinente- de comprender por qué ninguno de estos conceptos aparece en $R c$. Por contrapartida, si se parte de que $R c$ y Erc son dos obras con enfoques y lenguajes distintos, se abre un camino más alentador para ponderar la magnitud de sus diferencias.

\section{Ciencia y sociedad: un horizonte lejano}

En $R c$, Kuhn defiende una amplificación de la unidad de análisis de la historia de la ciencia, consistente en sustituir la concepción restringida de la bistoria de las teorías astronómicas, por una idea más amplia de historia intelectual de las ideas científicas, que incluye entre otras cosas la conceptualización del impacto de la ciencia en campos extracientíficos. Asoma aquí una divergencia importante con Erc, ya que tanto la noción de paradigma como la tesis del aislamiento de las comunidades científicas, conduce a que en Erc la unidad de análisis quede restringida a las ideas científicas y a la práctica científica, no en el sentido limitado que se rechaza en $R c$, pero sí en un sentido que vuelve secundarias tanto las motivaciones como las consecuencias sociales del cambio científico, que sí resultan de interés en trabajo de 1957.

El impacto social de los descubrimientos científicos, las condiciones prácticas que los impulsan, la función social de las cosmologías, y las prácticas sociales relacionadas con la observación de los cielos, son sólo algunos de los integrantes de una variada familia de procesos externos a la ciencia, que en $\mathrm{R} c$ son puestos de relieve bajo esta concepción historiográfica amplia, y que no encontrarán lugar en el enfoque más restringido de Erc. Dice Kuhn en la primera, haciendo alusión a los pueblos de la antigüedad: "Veneraban al Sol como a un dios y, a un mismo tiempo, le consideraban como el guardián del calendario, indicador de facto del ciclo seguido por las estaciones, del que dependían sus trabajos agrícolas." (1957, pp. 34-35/10). Puede verse que la delimitación amplia 
del pensamiento cosmológico -científico y extracientífico- como unidad de análisis de $\mathrm{R} c$, comporta un interés manifiesto por épocas en que las creencias cosmológicas aún no habían cristalizado en tradiciones científicas maduras, así como en la funcionalidad de dichas creencias respecto a rasgos específicos de las estructuras sociales.

En Erc por el contrario, el mundo social más allá de la ciencia parece convertirse en un horizonte cada vez más lejano. El interés por la prehistoria de las disciplinas científicas -vgr.-, está presente sólo de modo circunstancial, ya que únicamente interesa como caso de contraste de las épocas paradigmáticas: "Sólo de tarde en tarde, como ocurre en los casos de la estática, la dinámica, o la óptica geométrica antiguas, los hechos recogidos con tan escasa guía de las teorías preestablecidas hablan con la suficiente claridad como para permitir que surja un primer paradigma." (Kuhn, 1962, p. 123/16).

En Erc el interés de Kuhn por las épocas pre-paradigmáticas se restringe a identificar las diferencias entre el modo de funcionamiento científico que instala un paradigma y el modo de funcionamiento previo, que en sentido estricto no cuenta como científico. El tratamiento de casos como la teoría eléctrica antes de Franklin o las historias naturales en la antigüedad -ambos en el cap. II de Erc-, muestra que su principal interés es dar cuenta de que una vez surgido el paradigma fundacional de una disciplina, se inaugura una forma de organización del trabajo científico que no tiene antecedentes en la prehistoria del campo. Ello contrasta con el interés de $\mathrm{R} c$ por los estadios prehistóricos de la observación astronómica, que no sólo son entendidos como materia genuina para la reconstrucción histórica, sino que son analizados a efectos de detectar rasgos comunes con la astronomía científica.

Algo similar ocurre con la función social de las creencias cosmológicas, asunto que ocupa un lugar de menor relieve aún, en nombre del aislamiento de las comunidades científicas y las constricciones derivadas de una unidad de análisis más estrecha, como son los paradigmas. La falta de relevancia de los aspectos externos se ampara en la tesis del aislamiento de las comunidades científicas, de la que no hay rastro en $R c$, y que da cuenta de cómo Kuhn entenderá el: "aislamiento sin parangón de las comunidades científicas maduras respecto de las exigencias de los ciudadanos y de la vida diaria. Tal aislamiento nunca ha sido completo, se trata más bien de una cuestión de grado. Sin embargo, no hay otras comunidades profesionales en las que el trabajo creador individual 
se dirija y se evalúe de manera tan exclusiva por otros miembros de la profesión." (1962, pp. 330-331/164)

Respecto de la reconstrucción histórica, esta tesis implica que la dinámica interna de funcionamiento de las comunidades -organizadas en paradigmas y tradiciones normales- proporciona un marco suficiente para explicar el desarrollo histórico de la ciencia, lo que deja en un rol secundario tanto a las influencias externas de la ciencia como a la aplicación práctica de los descubrimientos científicos. Paul Hoyningen-Huene advirtió a este respecto que "debemos simplemente recordar que la teoría de Kuhn se orienta solamente a la ciencia 'pura', y entonces procede bajo la asunción de que la ciencia pura y la aplicada pueden ser distinguidas.” (1993, 6)

\section{La oposición al copernicanismo}

En escritos posteriores a Erc Kuhn reafirma esta concepción historiográfica que otorga autonomía explicativa al desarrollo de las comunidades científicas como unidad de análisis, frente a la influencia -siempre secundaria- de los factores extracientíficos. A este respecto, en "Las relaciones entre la historia y la historia de la ciencia", publicado originalmente en 1971 e incluido en $\mathrm{La}$ tensión esencial, afirma que "los historiadores, invariablemente, parecen dar excesiva importancia al papel del clima exterior en las ideas extracientíficas... el medio en que se da la actividad intelectual reacciona sobre la estructura teórica de una ciencia únicamente en la medida en que lo ameritan los problemas técnicos concretos a los que se enfrentan los profesionales de ese campo." (1977, pp. 161-162/137-138)

Si bien no es nuestro propósito analizar el curso del pensamiento historiográfico de Kuhn luego de 1962, cabe sí señalar que el tono general de los ensayos historiográficos contenidos en La tensión esencial, prolonga las orientaciones predominantes en Erc, tanto en lo que hace al aislamiento de las comunidades científicas como a la insuficiencia explicativa de los factores externos para las revoluciones científicas ${ }^{14}$. Asimismo, en los pocos pasajes de

14 Un estudio detallado de los textos contenidos en La tensión esencial merecería un trabajo aparte, más cuando nuestro período de análisis abarca preferentemente el período 19571962. Sin embargo, podemos arriesgar la idea de que el tono general de los estudios

Manuscrito - Rev. Int. Fil., Campinas, v. 38, n.3, pp. 25-57, set.-dez. 2015. 
La tensión esencial en que Kuhn hace referencia a $R c^{15}$, se mantiene -como ya señalamos en relación al Prefacio- la postura de que el estudio de 1957 es un caso ilustrativo de las tesis historiográficas del Kuhn maduro.

Por contraste a esto, un ejemplo del enfoque amplio que Kuhn maneja en 1957 es la reconstrucción del pasaje del universo finito de Copérnico al universo infinito de Newton. Para explicar este proceso en $\mathrm{R} c$, Kuhn apela reiteradamente a las cosmologías de Nicolás de Cusa y Giordano Bruno, como contribuciones a la idea del universo infinito, asociadas -una como antecedente y la otra como derivación- a la obra de Copérnico. Es interesante destacar cómo Kuhn subraya que ni los aportes de Cusa ni los de Bruno cuentan como científicos $^{16}$, en cuanto se trata de especulaciones filosóficas distantes del trabajo profesional de la astronomía, incluso en el caso de Cusa, a quien considera "un místico que rechazaba alegremente las apariencias a favor de una aprehensión trascendente de la divinidad infinita en la que todas las paradojas se reconciliaban." (Kuhn 1957, p. 303/235) ${ }^{17}$

El peso explicativo atribuido a ambos filósofos en el desarrollo de la revolución copernicana es coherente con el programa historiográfico que Kuhn maneja por 1957, que busca reconstruir el desarrollo de la ciencia considerando la influencia de las ideas extracientíficas en el surgimiento de nuevas teorías. Westman ha destacado con precisión que en la narrativa de $R c$ el principal frente de oposición al esquema conceptual copernicano no proviene de los astrónomos profesionales, sino de agentes externos a la práctica de la disciplina: "la batalla sobre la nueva teoría ocurrirá entre aquellos que están dentro y aquellos que están

historiográficos de La tensión esencial, sigue de cerca los presupuestos de Erc, con la consiguiente distancia -si estamos en lo correcto- respecto de la historiografía de $\mathrm{R} c$.

${ }^{15} \mathrm{El}$ que acabamos de consignar es uno de ellos. La segunda referencia aparece en "La función de la medición en la física moderna”, de 1961, en relación a las discrepancias entre los sistemas de Copérnico y Ptolomeo (Kuhn, 1977, pp. 209/184).

${ }^{16}$ De modo similar a la consideración de personajes como Bacon o Plinio en Erc (1962, cap. II).

${ }_{17}$ A este respecto Westman (1994, p. 87), apunta que el papel preponderante que Kuhn le atribuye al neoplatonismo como impulsor de la revolución copernicana, proviene de una lectura acrítica de la obra de E. A. Burtt, Metaphysical Foundations of Modern Physicial Science, publicada en 1925.

Manuscrito - Rev. Int. Fil., Campinas, v. 38, n.3, pp. 25-57, set.-dez. 2015. 
fuera del espacio de especialización de la astronomía técnica. La oposición venía de los 'outsiders': teólogos como Martín Lutero, John Calvin, y Philipp Melanchthon; el filósofo político Jean Bodin; los poetas Guillaume Du Bartas y John Donne." (Westman 1994, p. 94).

Compárese la cautelosa y justificada renuencia de Westman a conceder a Kuhn que la oposición al copernicanismo se centraba al exterior de la ciencia, con la exaltada defensa que sobre el punto efectúa N. Swerdlow: "y fue siempre la religión, desde el comienzo, cuando Lutero llamó a Copérnico un bufón, hasta la condena de la iglesia en 1616, y hasta el siglo XVII cuando los clérigos en las órdenes continuaban probando que la tierra no se movía, mucho tiempo después de que Kepler, Galileo y Newton presentaran evidencia abrumadora de que lo hace. Y Kuhn puso su dedo en ello directamente, en doce de las mejores páginas que he leído sobre el tema." (Swerdlow 2004, p. 93)

Como contracara de esto, Westman (1994, p. 111) recuerda que los astrónomos jesuitas -cuyo centro de operaciones era el observatorio Vaticano ${ }^{18}$ fueron desde principios del siglo XVII el principal frente de oposición tanto a Kepler como a Galileo, y que no juegan ningún papel relevante en la reconstrucción kuhniana de $\mathrm{R} c^{19}$. La no consideración de este grupo lleva a Kuhn (1957, pp. 293-295/226-228) a afirmar que para la segunda mitad del siglo XVII no había oposición científica al copernicanismo en Europa, lo cual es puesto en cuestión por el trabajo de los jesuitas, particularmente de Giovanni Battista Riccioli (1598-1671) quien presentó una elaborada defensa del sistema geocéntrico en su Almagestum Novum de 1651 (Feingold, 2003).

Curiosamente, el papel tanto de Christopher Clavius como de Riccioli en la polémica con el heliocentrismo sí es destacado por uno de los autores de referencia de Kuhn, A. Rupert Hall en La revolución cientifica (Rupert Hall, 1954); pero no hay mención de este grupo y de su papel en la revolución copernicana

18 Algunos de los principales astrónomos jesuitas del observatorio vaticano, acérrimos defensores del geocentrismo, fueron Christopher Clavius (1538-1612), Christoph Grienberger (1561-1636), Giuseppe Biancani (1566-1624), Giovanni Paolo Lembo (1570-1618) y Mario Bettinus (1582-1657).

${ }^{19}$ Una cierta rehabilitación de la oposición jesuita a Galileo se intenta en Fischer (1980). Tratamientos de la oposición al copernicanismo, que dan por sentado lo dicho por Kuhn sobre el estado de la cuestión en la segunda mitad del s. XVII, pueden encontrase en Swerdlow (2004, pp. 91-93) y Marcum (2005, p. 40-41). 
en ninguna de las dos obras de Kuhn. Como señala Westman, la omisión no es llamativa en el caso de $R c$, en cuanto en esta obra el frente de oposición al copernicanismo se ubica al exterior de la ciencia; pero sí lo es en el caso de Erc, ya que entre otros episodios, el tono de la disputa entre los jesuitas y Galileo sobre los descubrimientos telescópicos del Sidereus Nuncius, encaja bastante bien en la idea kuhniana del cambio conceptual como enfrentamiento entre tradiciones inconmensurables.

Por otra parte, no es extraño que Giordano Bruno y Nicolás de Cusa no aparezcan nombrados ni una sola vez en Erc, lo mismo que Melanchthon, Lutero o Calvino, de mención frecuente en $\mathrm{R} c$. De acuerdo a las diferencias de dominio de análisis que hay en ambas obras, los frentes de oposición externos jerarquizados en $R c$ juegan un papel lateral en la dinámica interna de lo que luego tomará el nombre de cambio paradigmático. Esto seguramente responde a que en Erc "el internalismo está presupuesto, al menos como una hipótesis fuerte de trabajo. Esto no excluye la influencia ocasional de factores externos pero implica que ésta es la excepción a la regla." (Hoyningen-Huene, 2012, p. 288)

Así es que en Erc los factores que explican la transición entre paradigmas remiten básicamente a los científicos que deciden asumir o rechazar un paradigma determinado, en medio de procesos intrínsecos a las comunidades científicas, que no pueden ser explicados ni reducidos a elementos no científicos, secundarios en relación a los problemas disciplinares que impulsan el cambio de paradigma. Pensamos que esta diferencia en el alcance de la unidad de análisis permite asimismo dar cuenta de la completa ausencia del término 'comunidad científica’ en la obra de 1957.

\section{Un papel para la historia interna}

Siguiendo esta línea, el Kuhn de $\mathrm{R} c$ finalmente sintetiza el triunfo del copernicanismo en tres niveles, en los que la nueva cosmología tuvo tiempos diferentes de imposición: 1. la comunidad de astrónomos profesionales, que para fines del siglo XVII ya se había convertido casi completamente; 2. las comunidades universitarias, en las que se enseñó el sistema de Copérnico junto al de Ptolomeo y Tycho Brahe, hasta entrado el siglo XVIII; 3. la cosmología popular, que abraca el conjunto de la sociedad no instruida en ciencia, donde el copernicanismo sólo se impuso a finales del siglo XVIII. 
Este triple nivel de análisis no es aplicable ${ }^{20}$ a las revoluciones científicas de Erc, en cuanto las transiciones entre paradigmas se desarrollan casi exclusivamente a nivel de las comunidades científicas que los adoptan. Procesos históricos como la expansión de un paradigma en las comunidades universitarias ajenas a su disciplina de origen, o la imposición de un paradigma a nivel social, son temas que no ocupan espacio alguno en 1962. Esto responde a la concepción historiográfica que Kuhn defiende por esta época, y que Paul Hoyningen-Huene denominó como nueva historiografía interna de la ciencia; si bien ésta no niega completamente la relevancia de los factores externos, mantiene a los factores internos como base explicativa de las revoluciones científicas "Mientras que el contenido sustantivo de las ciencias maduras está ampliamente aislado de la influencia de factores externos, no está tan desafectado como la mayoría de los autores de orientación exclusivamente internalista sugerirían." (HoyningenHuene, 1993, p. 19) 21

Aquí podría pensarse que hay sólo una diferencia de énfasis y de objetivo, de forma que partiendo de la existencia de tres niveles, Erc se ubica en uno de ellos sin desconocer los otros dos. Pero el problema es de fondo. Erc pretende ser una explicación más bien exhaustiva de por qué se producen las revoluciones científicas, y en la medida en que articula una imagen del cambio revolucionario que enfatiza los procesos internos a los paradigmas y deja de lado

${ }^{20}$ Podríamos decir que este triple nivel de análisis no es aplicado por Kuhn en 1962, pero ello únicamente alude a una decisión de facto, que sólo tendría el valor de describir lo que Kuhn decidió no hacer. Por el contrario, al afirmar que este triple nivel de análisis no es aplicable a Erc, pretendemos dar cuenta no de una decisión fáctica, sino de una limitación conceptual: Kuhn no aplicó el triple nivel de análisis en Erc, ni tampoco podría haberlo aplicado, en cuanto la matriz historiográfica que sostiene la distinción entre los tres niveles de las revoluciones científicas no es asimilable con la historiografía de Erc.

${ }^{21}$ Es de hacer notar que la amplia reconstrucción de la historiografía Kuhniana que emprende Hoyningen-Huene (1993), parte de Erc y se prolonga hacia los escritos posteriores, prestando escasa o nula atención al pensamiento historiográfico de Kuhn en la década de los 50's -lo mismo sucede en Hoyningen-Huene (2012) y Bird (2015)-. En este sentido, coincidimos plenamente con Hoyningen-Huene respecto a los rasgos de la 'historiografía interna' que Kuhn maneja en obras como Erc y La tensión esencial, pero con la salvedad de que si prolongamos el análisis hacia atrás en el tiempo, la historiografía de $\mathrm{R} c$ no resulta asimilable a este modelo internalista.

Manuscrito - Rev. Int. Fil., Campinas, v. 38, n.3, pp. 25-57, set.-dez. 2015. 
los factores sociales, la eficacia explicativa atribuida a unos y quitada a otros no puede reducirse a una cuestión de propósito, sino a la premisa subrayada por Hoyningen-Huene de que el 'contenido sustantivo' del desarrollo científico puede explicarse solamente mediante factores internos.

$\mathrm{El}$ análisis centrado en comunidades científicas, paradigmas y tradiciones normales, es casi totalmente asimilable al nivel 1. de $R c$, e incluso en las consideraciones sobre el papel de la educación científica que Kuhn efectúa en el capítulo XI de Erc puede verse una familiaridad parcial con el nivel 2., que refiere a la enseñanza universitaria. Pero esto no quita que en E $r$ lo sustantivo de los niveles 2. y 3. está fuera del foco de interés, en cuanto el concepto de paradigma busca dar cuenta de cómo las comunidades científicas se definen -y acaso se clausuran-, en torno a determinadas creencias no necesariamente compartidas por los legos. Esto hace que problemas como el ritmo de imposición de un paradigma fuera de la comunidad científica, y las vicisitudes que atraviesa en otros ámbitos, no tengan relevancia explicativa en el marco de Erc.

La diferencia de enfoque en ambas obras puede verse también cuando en $R c$ se afirma "nuestro interés no se centrará tanto en la propia astronomía como en el medio ambiente intelectual y, en el ámbito social y económico en cuyo seno se practicaban los estudios astronómicos." (1957, p. 25/3). Es de recibo señalar que en Erc también hay cabida para factores de tipo psicológico y sociológico, pero éstos operan en la interna de las comunidades científicas -más específicamente en los procesos de cambio paradigmático- y no en el ámbito social o económico que da contexto al trabajo científico. El enfoque historiográfico de $R c$ apunta a una comprensión amplia de la ciencia en su contexto y en sus implicancias sociales, lo que lleva a Kuhn a incluir como factores relevantes para el entendimiento de la revolución copernicana la decadencia del Imperio Romano, las invasiones árabes a Europa, la conformación del feudalismo medieval, la fundación de las universidades europeas y la necesidad de reformar el calendario en el siglo XVI, por sólo mencionar algunas ${ }^{22}$.

La mayoría de estos procesos sociales y extracientíficos son mencionados sólo al pasar en el análisis de la revolución copernicana de Erc, y

\footnotetext{
22 Sobre la importancia de la reforma del calendario en la revolución copernicana afirma: "La necesidad de llevar a cabo una reforma del calendario constituyó una importante causa motriz de la propia reforma de la astronomía...” (1957, p. 36/11).
}

Manuscrito - Rev. Int. Fil., Campinas, v. 38, n.3, pp. 25-57, set.-dez. 2015. 
Kuhn matiza fuertemente su papel como elementos causales de las revoluciones científicas:

Naturalmente, el fallo de la actividad normal de resolución técnica de problemas no fue el único ingrediente de la crisis astronómica a la que se enfrentó Copérnico. Un tratamiento amplio de la cuestión habría de incluir la presión social para la reforma del calendario... la crítica medieval a Aristóteles, el surgimiento del neoplatonismo renacentista, además de otros elementos históricos significativos. Con todo, el fracaso técnico seguiría siendo el núcleo de la crisis. En una ciencia madura, y la astronomía lo era ya desde la antigüedad, los factores externos del tipo de los mencionados antes son significativos sobre todo para determinar el momento del fracaso, la facilidad con que se reconoce y el área en que se produce por primera vez debido a que recibe allí especial atención. Aunque resulten enormemente importantes, este tipo de cuestiones cae fuera de los límites de este ensayo. (Kuhn, 1962, p. 198/69)

La última parte del pasaje puede nuevamente dar la impresión de que se trata sólo de una cuestión de enfoque, y que la diferencia se reduce a que en Erc Kuhn solamente no está interesado en los factores externos, a los que aún considera relevantes. Desde este punto de vista, $R c$ apunta a la reconstrucción de una revolución científica particular, mientras que Erc busca articular ciertas tesis histórico-filosóficas sobre la naturaleza de las revoluciones científicas. La primera empresa requiere un análisis de los factores sociales, y la segunda no; diríamos entonces que Erc reconoce los factores sociales jerarquizados en $\mathrm{R} c$, pero no los incorpora a la reconstrucción histórica. Una historia completa de la revolución copernicana debería incluir tanto la dinámica interna de las comunidades científicas, como los factores externos propios del mundo social, que es lo que encontramos al conjuntar los dos libros ${ }^{23}$.

${ }^{23}$ No podemos atribuir literalmente este argumento a ninguno de los referentes de lo que hemos denominado perspectiva continuista de la obra de Kuhn, Heilbron (1998), Swerdlow (2004) y Marcum (2005), ya que ninguno de sus trabajos incluye una consideración comparativa de los factores internos y externos en los dos primeros libros de Kuhn. Cabe pensar que si se da por supuesto que hay un único proyecto filosófico que va de una obra a otra, la comparación misma pierde sentido. La cuestión de lo interno y lo externo en ERC sí es objeto de comentario en la nota de Bravo (1979) y en el artículo de Westman (1994).

Manuscrito - Rev. Int. Fil., Campinas, v. 38, n.3, pp. 25-57, set.-dez. 2015. 
Entendemos que esta manera de encausar el asunto es inapropiada, ya que las diferencias van más allá de una cuestión de dominio o propósitos conceptuales. En el marco historiográfico de $R c$, la consideración de los factores externos es indispensable para la comprensión de las revoluciones científicas, por lo que una imagen del cambio revolucionario que considere estos factores como fuera de sus límites, mantiene con el marco historiográfico de $\mathrm{R} c$ algo más que un desacuerdo de objetivos. Esta diferencia es de fondo, y estriba en que la concepción historiográfica de Erc supone que la dinámica de funcionamiento de la ciencia puede explicarse plenamente sin necesidad de atribuir eficacia causal a los factores externos. Es de señalar que a texto explícito, sólo vemos en Kuhn una actitud cautelosa sobre este asunto. En el ya referido de La tensión esencial, señala que Erc

debe entenderse como un intento de explicar por qué la evolución de las ciencias más desarrolladas ha ocurrido con relativa independencia del medio social... leído de esta manera, el libro puede considerarse el primer paso para quienes tratan de adentrarse en el estudio de las formas que adoptan tales influencias externas, así como los cauces por los que discurren. (1977, p. 15/XV)

El mismo tono conciliador puede encontrarse en el prefacio de Erc, cuando afirma que la consideración de los factores externos en $\mathrm{R} c$ no afecta las tesis de $\operatorname{Erc}(1962$, p. 97/X). Sin embargo, la complementariedad entre lo interno y lo externo resulta más una expresión verbal que una postura historiográfica sostenible. Esto no sólo disimula el hecho de que Erc abraza una historiografía netamente interna, sino también impide detectar que los cambios en el pensamiento historiográfico de Kuhn desde 1957 a 1962 no pueden -quizás afortunadamente- integrarse en una unidad coherente. El mismo Paul Hoyningen-Huene debió admitir que "la integración sistemática de los factores internos y externos permanece más como un ideal que como la práctica estándar aún en la reciente historiografía de la ciencia." (Hoyningen-Huene, 1993, p. 19). Por lo que en el mejor de los casos, y tomando como referencia al más amistoso de los comentaristas de Kuhn, la integración entre lo interno y lo externo es antes un ideal a alcanzar que un rasgo consumado del periplo historiográfico de Kuhn.

Pero en una consideración más crítica del asunto, Carlos Solís, ha señalado que: "a pesar de sus tributos de boquilla a la importancia de la tecnología y otro factores externos, la verdad es que -Kuhn- nunca supo cómo combinar 
tales cosas con su visión internista, típicamente koyreana, según la cual lo esencial son las ideas...". (en Kuhn, 1962, pp. 81) No es nuestro propósito aquí determinar hasta qué punto Kuhn logró conjugar su visión internista con los factores externos, pero sí en algún momento de su obra estuvo cerca de hacerlo, ese momento fue en la década de los 50 's durante la elaboración de $R c$, ya que no a partir de la década de los 60's, momento en que entendemos se aplica de pleno derecho la ácida acusación de Solís.

Esto entra en cierto conflicto con el balance que el mismo Kuhn efectuó en 1995, cuando durante una entrevista recordó que a propósito de Erc, Alexander Koyré "decía: «Usted ha unificado la historia externa e interna de la ciencia, las cuales han estado muy alejadas en el pasado». Ahora bien, a mí no se me había ocurrido que fuera eso lo que había hecho. Entendí lo que quería decir, y que la observación viniera de él era particularmente satisfactorio..." (Kuhn, 1995, p. 333) No pretendemos aquí construir una exégesis conceptualmente densa a partir de una anécdota, no obstante un comentario de Kuhn a renglón seguido recae en la idea -con mención explícita a $R c$ - de que la diferencia de peso entre lo externo y lo interno es una cuestión de énfasis y no de fondo.

Inicialmente Kuhn reafirma su filiación internalista, pace los elogios de Koyrè: "Lo que quiero decir es que entendí a lo que se refería... Yo pensaba que el libro era claramente internalista. Los ingleses siempre se sorprenden de que yo sea un internalista. No consiguen entenderlo... Si miran la introducción de mi libro sobre la revolución copernicana, verán que en cierto modo me disculpo por la falta de consideración de muchos factores externos, y afirmo que si los hubiera considerado habría tratado más extensamente la importancia del calendario y otros factores de este tipo." (Kuhn, 1995, 334).

A pesar de lo críptico de estos pasajes, nos permitimos especular con que ‘la integración entre lo interno y lo externo’ a la que se refiere Koyré responde a que Kuhn incorpora los factores psicológicos propios de cada científico -como el caso del culto al Sol en Copérnico o Kepler-, así como aspectos sociológicos del funcionamiento de las comunidades científicas. Pero como ha señalado Alexander Bird (2015) estos factores -que entendemos podrían considerarse 'externos' bajo la mirada de Koyré- siguen estando inscriptos en la dinámica interna del funcionamiento de las comunidades científicas, y no califican como el tipo de influencia social que la historiografía externa le adscribe a la ciencia. Como ya señalamos, esto responde a que Erc no sólo se basa sino 
que requiere una historiografía interna, para llevar a buen puerto sus propósitos de reconstrucción de la ciencia.

Por otra parte, el balance que Kuhn efectúa de Rc en 1995 sigue siendo una suerte de flirteo con la historiografía externa de la ciencia -en la que su obra resultó tan influyente-, pero entendemos que en el camino quedan confundidos dos niveles. Por un lado, es de recibo que los factores externos podrían haber tenido aún más relevancia en $R c$, y ello es posible sólo porque esta obra supone que tanto las causas como las consecuencias de las revoluciones científicas responden a la interacción entre los factores internos y los externos. Pero el mismo razonamiento no se aplica a Erc, cuyo curso narrativo no bubiera permitido dar más relevancia a los factores externos, en cuanto supone una historiografía para la cual es necesario que el funcionamiento histórico de la ciencia pueda ser explicado básicamente por factores internos.

La perspectiva historiográfica de Erc busca identificar los rasgos estructurales de funcionamiento de la ciencia y los patrones de comportamiento definitorios de las comunidades científicas, lo que exige definir unidades de análisis -paradigmas, comunidades científicas- que permitan dar cuenta de la especificidad de la ciencia más allá de las variables de contexto y las influencias sociales. Para Bird (2015), esto responde a que la imagen kuhniana de la ciencia normal como actividad basada en la resolución de rompecabezas -cuya relevancia, contenido y métodos de resolución son definidos por las comunidades científicas- exige la adopción de un enfoque internalista del desarrollo científico. En esta medida, si bien los factores externos pueden influir en la forma en que ocurre una revolución científica -como el caso de la reforma del calendario en la revolución copernicana-, son los factores internos los que a fin de cuentas explican por qué ocurrió la revolución.

\section{Revoluciones científicas y cambios de mundo}

La reformulación que experimenta el pensamiento de Kuhn entre 1957 y 1962 también se aprecia en la forma en que un mismo grupo de conceptos aparece en cada época tratado bajo ópticas diferentes. En Rc Kuhn incorpora los desarrollos de la psicología de la Gestalt en apoyo de su reconstrucción histórica, para subrayar cómo las pautas culturales moldean los procesos perceptivos, "no... debe descartarse la posibilidad de que el pastor o el navegante de la 
antigüedad, al escrutar hora tras hora los cielos estrellados, 'viera' realmente dibujada por las estrellas las figuras mitológicas que le eran familiares, del mismo modo que nosotros 'vemos' algunas veces rostros en las nubes o en los contornos de los árboles. Las experiencias de la moderna psicología de la gestalt subrayan la universal necesidad de descubrir esquemas familiares en agrupaciones aparentemente debidas al azar...” (1957, p. 39/14).

También la configuración gestáltica de los procesos perceptuales es relevante en Erc, donde las revoluciones científicas se explican por una suerte de cambio de gestalt o conversión que conlleva una nueva forma de ver el mundo bajo los códigos del nuevo paradigma 24 . En Erc afirma "Las demostraciones conocidas de los cambios en la Gestalt visual resultan muy sugerentes en cuanto prototipos elementales de estas transformaciones del mundo de los científicos. Lo que antes de una revolución eran patos en el mundo científico, son conejos después de ella." (Kuhn 1962, pp. 256-257/111)25.

Los desarrollos de la Gestalt a los que Kuhn recurre son los mismos en ambos casos, lo que permite entrever su temprano interés por los procesos psicológicos involucrados en la adopción de las creencias científicas. Sin embargo, a pesar de la presencia de los conceptos gestálticos en ambas obras, el dominio al que se aplican en cada caso es bien diferente. En $\mathrm{R} c \mathrm{Kuhn}$ utiliza el aparato de la Gestalt buscando dar cuenta a modo general, de la determinación cultural de los procesos perceptivos -dentro y fuera de la ciencia-, mientras que en Erc la teoría gestáltica funciona como una analogía para comprender la forma específica en que los paradigmas moldean la percepción de la comunidad científica. En el primer caso se trata de procesos intrínsecos a la relación entre los conceptos culturales y el mundo, de los que la ciencia es una entre otras expresiones. En el segundo se trata de procesos singulares relativos a cómo un

\footnotetext{
${ }^{24}$ Robert Westman ha señalando este como uno de los rasgos fundamentales de la nueva historiografía que Kuhn adopta en 1962: "La nueva especie de momento revolucionario de Kuhn en ERC tiene sobre sí un timbre antimodernista: los científicos ahora deciden, sin una justificación completamente razonada, cambiar sus creencias y compromisos a causa de un cambio perceptual irreversible, similar a una experiencia de conversión." (Westman 1994, p. 85)

25 Sobre el contenido y las implicancias de la noción kuhniana de cambio de mundo, son de relieve los trabajos de Hoyningen-Huene (1993), Pérez Ransanz (1999) y Bird (2000).
}

Manuscrito - Rev. Int. Fil., Campinas, v. 38, n.3, pp. 25-57, set.-dez. 2015. 
paradigma estructura la percepción de los científicos, que no necesariamente tienen un correlato en los procesos perceptivos dados fuera de la ciencia.

Este carácter diferencial de la percepción científica respecto a la percepción ordinaria -objeto inicial de los estudios gestálticos-, es explícitamente subrayado por Kuhn en 1962, en un tono que reivindica la especificidad cognitiva de la ciencia, afirmando que los experimentos de la Gestalt. "Muestran características de la percepción que podrían ser centrales en el desarrollo científico, pero no demuestran que las observaciones precisas y controladas realizadas por los científicos que investigan compartan en absoluto esas características. " (Kuhn, 1962, p. 260/113)

La base de esta divergencia está en que la visión de la ciencia que Kuhn ofrece en $R c$, supone una continuidad entre la percepción científica y la percepción fuera de la ciencia, lo que da a pensar que la psicología de la Gestalt ilustra aspectos relevantes de todo proceso perceptual -sea científico o no-. Pero en el enfoque de Erc, mucho más centrado en la singularidad de la ciencia y menos permeable a la continuidad con los elementos culturales, la eficacia de la Gestalt en dar cuenta de los procesos perceptivos extracientíficos, no asegura que la percepción científica pueda ser explicada de igual manera. Esto en cuanto para 1962 Kuhn parece entender que la percepción científica es un proceso sumamente peculiar, cuyo funcionamiento no puede asimilarse completamente al de la percepción extracientífica.

A partir de aquí puede entreverse otro aspecto el uso de la Gestalt en ambas obras, vinculado a la cuestión del cambio de mundo, asociada en Erc a la conversión que experimentan los científicos al adoptar un nuevo paradigma. Para Floris Cohen (1994, pp. 123-124), la idea de que las revoluciones científicas conllevan un cambio de cosmovisión toma forma en Kuhn como resultado de la influencia de The Origins of Modern Science, 1300-1800, de Herbert Butterfield, publicada en 1949. Aunque es necesario señalar que en la única mención a la obra en Erc Kuhn toma explícita distancia de Butterfield ya que su postura implicaba que después de una revolución, los científicos ven lo mismo pero lo interpretan de forma diferente, justamente la concepción que Kuhn comienza a combatir en 1962. Sin embargo la idea -claramente más afín a Butterfield- de que el cambio de esquemas conceptuales es un cambio de interpretaciones sobre un contenido empírico estable, resulta sí central en el Kuhn de $\mathrm{R} c$, por lo que si el trabajo de Butterfield ejerció algún tipo de influencia sobre Kuhn -como defiende Cohen y Kuhn reconocerá sólo posteriormente (1977, p. 13/12)-, esta influencia 
corresponde más a 1957 que a 1962, en cuanto por esta época Kuhn ya niega explícitamente la historiografía de The Originis of the Modern Sciencere.

Westman por su parte, ha señalando que la imagen del cambio teórico como conversión está estrechamente ligada a la tesis de la inconmensurabilidad, lo que explica su falta de desarrollo en $\mathrm{R} c$, y el hecho de que en su primer libro Kuhn no produzca historias de conversión sobre ninguno de los personajes relevantes de la revolución copernicana (1994, pp. 94-95), en la misma medida en que no produce historias de inconmensurabilidad ${ }^{27}$. Por el contrario Marcum, ha asumido que el cambio de mundo responde a una misma conceptualización en Rc y Erc. Refiriéndose inicialmente a la primera, afirma: "la revolución de Copérnico cambió la manera en que el mundo es visto no sólo por los astrónomos, sino también por los no astrónomos. Este cambio de mundo, como después indicó $K u b n^{28}$, era el resultado de un conjunto de nuevos desafíos, nuevas técnicas, y nuevas formas hermenéuticas de interpretar los datos." (2005, p. 42).

Frente a esta perspectiva continuista, el argumento de Westman resulta eficaz: la expresión 'cambio de mundo' no puede significar lo mismo en las dos obras de Kuhn, porque en Erc aparece ensamblada a un arsenal de conceptos conversión, inconmensurabildiad, variación de significado- que están ausentes en $R c^{29}$. Pero además, también cabe señalar que en $R c$ el cambio de visión del mundo implica literalmente que un nuevo esquema conceptual interpreta de forma diferente los mismos datos ya conocidos por el esquema anterior, en cuanto un esquema no es más que un marco interpretativo de un conjunto de datos que se

${ }_{26}$ El trabajo de Cohen -como muchos otros escritos sobre el tema- se propone reconstruir el pensamiento historiográfico de Kuhn tomando como base Erc y trabajos posteriores, sin hacer referencia alguna al libro de 1957.

${ }^{27}$ Una revisión pormenorizada de las relaciones entre inconmensurabilidad y cambio de mundo se halla en Grandy (2003), un tratamiento reciente de la tesis de la inconmensurabilidad se encuentra en Gentile (2013).

${ }^{28} \mathrm{El}$ destacado es nuestro, ya que el 'después' alude a Erc.

${ }^{29}$ La única vía para neutralizar esto, sería negar que en $\mathrm{R} c$ esté del todo ausente la tesis de la inconmensurabildiad, posibilidad algo escabrosa de defender, y que Marcum sólo desliza elípticamente: "La visión de Kuhn sobre cómo leer un texto de una era científica anterior también iba a formar la columna vertebral de la tesis de la inconmensurabilidad." (2005, p. 9) 
mantiene estable. Dice Kuhn: "consideraremos las observaciones estrictas antes de explicarlas dentro de un marco interpretativo. Una vez más, el conflicto entre diversas interpretaciones nos situará ante un problema nuevo y fundamental de la anatomía de la creencia científica." (1957, p. 76/45)

En Erc por el contrario, la noción de cambio de mundo se introduce en un contexto que lleva a cuestionar la permanencia de los datos y la pertinencia de la interpretación como operación básica para explicar las revoluciones científicas: "los datos no son inequívocamente estables. Un péndulo no es una piedra que cae y el oxígeno no es aire desflogistizado. Por consiguiente, los datos que los científicos recogen de estos diversos objetos, son ellos mismos distintos...". (Kuhn, 1962, p. 271/121) Hoyningen Huene introdujo una interpretación neokantiana de este punto, señalando que lo que 'cambia' es el mundo fenoménico, cuyos límites y rasgos fundamentales están determinados por las estructuras conceptuales de las comunidades científicas. Al mismo tiempo, lo que no cambia es 'el mundo en sí', del que no tenemos conocimiento directo, y al que sólo accedemos parcial e inferencialmente a través de nuestras teorías. La diferencia estriba en que para Kant a un único mundo noumenal -en sícorresponde un único mundo fenoménico, mientras que para Kuhn hay -a lo largo de la historia- varios mundos fenoménicos diferentes, frente al único mundo en sí, lo que da lugar a la tesis de la pluralidad de los mundos fenoménicos (1993, p. 31 y ss.).

Por el contrario, en el marco de $R c$ "Cada observación es una pieza aislada en una larga lista de hechos brutos - bare facts-30 que hacen referencia a los cielos..." (1957, p. 67/37), lo que permite considerar el enfrentamiento entre esquemas conceptuales rivales como resultado de diferencias interpretativas respecto de una base de 'hechos brutos' relativamente estable. A la luz de esto, el marco de $R c$ no deja espacio conceptual para introducir algo como la tesis de la pluralidad de mundos fenoménicos detectada por Hoyningen-Huene en Erc, en cuanto el único mundo fenoménico que acepta $R c$ es la plataforma sobre la que posteriormente se despliegan las diferencias entre los esquemas conceptuales rivales. Siguiendo la afirmación de Hoyningen-Huene $(2012,281)$ de que toda historiografía supone elementos filosóficos, podemos tomar este caso como una muestra de la divergencia entre la historiografía de Rc y la de Erc.

30 También puede traducirse como 'hechos puros', para dar cuenta de la consideración que por esta época Kuhn tiene respecto de la base empírica de las teorías científicas.

Manuscrito - Rev. Int. Fil., Campinas, v. 38, n.3, pp. 25-57, set.-dez. 2015. 
Este recorrido permite entrever que el tratamiento de la concepción gestáltica de la visión en $\mathrm{R} c$ sólo se aplica a los aspectos perceptuales del conocimiento, sin que puedan atisbarse las revulsivas consecuencias epistemológicas y ontológicas que se extraerán en Erc. De esta forma, los mismos conceptos psicológicos que en 1962 llevarán a Kuhn a hablar de conversión, cambio de mundo e incomunicabilidad entre paradigmas ${ }^{31}$, en 1957 sólo dan base a la idea mucho menos escandalosa de que la percepción está moldeada por las herramientas conceptuales, el contexto cultural y las expectativas previas de los sujetos.

\section{Historiografía casuística vs. historiografía estructural}

En el marco de $R c$, es explícita la idea de que la revolución copernicana muestra aspectos excepcionales del cambio científico, solamente presentes en otros pocos casos como los de Freud, Darwin o Einstein ${ }^{32}$, y que parte de su carácter excepcional está dado por su impacto en diferentes niveles de la vida social: "en lo que respecta a sus consecuencias extra-científicas, la teoría copernicana no puede ser considerada como típica, pues pocas han sido las teorías científicas que han desempeñado un papel tan importante en el marco del pensamiento no científico." (Kuhn, 1957, p. 27/4). En Erc mientras tanto, el propósito de Kuhn se limita a utilizar la revolución copernicana -entre otras revoluciones- para mostrar algunos rasgos intrínsecos a la ciencia, que en mayor o menor medida se dan en toda revolución científica, en cuanto procesos estructurales de cambio paradigmático. Luego si estas revoluciones tienen consecuencias sociales más o menos impactantes, más o menos inmediatas,

31 "Por consiguiente, en tiempos revolucionarios, cuando cambia la tradición de la ciencia normal, la percepción que tiene el científico de su medio ha de reeducarse; en algunas situaciones familiares, ha de aprender a ver una nueva Gestalt. Una vez que lo haya hecho, el mundo de su investigación parecerá ser aquí y allá inconmensurable con el que habitaba antes." (Kuhn, 1962, p. 257/112)

32 Es de notar que Darwin y Einstein se mantienen como ejemplos de revoluciones en Erc, cosa que no sucede con Freud, seguramente en razón del status preparadigmático que la mayoría de las ciencias sociales terminan teniendo en el pensamiento de Kuhn.

Manuscrito - Rev. Int. Fil., Campinas, v. 38, n.3, pp. 25-57, set.-dez. 2015. 
constituye un área de problemas que no es prioritaria en el marco de análisis de Erc.

Cuando Kuhn señala que la unidad de análisis de $\mathrm{R} c$ es el "pensamiento cosmológico" (1957, pp. 27-28), ello muestra por un lado que ya había recorrido suficiente camino como para dejar atrás la "historia de las teorías astronómicas", pero aún no había alcanzando el grado de desarrollo de su pensamiento necesario para dar forma a un dominio de análisis situado más allá de la especificidad del caso histórico. Justamente esto es lo que va a irrumpir en Erc, en cuanto en 1962 el análisis histórico de la revolución copernicana revelará la misma estructura que el análisis de otras revoluciones científicas; mientras que en $\mathrm{R} c$ es vista como una revolución altamente singular, dotada de características muy peculiares que en cierto punto justifican su análisis histórico; en Erc por el contrario, se pierde el carácter excepcional de la revolución copernicana.

De esta forma, en $R c$ la reconstrucción histórica de la revolución copernicana es un fin en sí mismo, mientras que en Ercoficia sólo como un caso que proporciona materia prima para un aparato filosófico de mucho más amplio alcance. Así, Kuhn pasa de la metodología historiográfica según la cual cada revolución científica es un objeto de análisis por derecho propio, a la idea muy diferente de que el estudio de cada revolución científica revela una estructura común a todas ellas. En estos términos, puede afirmarse que $R c$ supone una bistoriografia casuística, mientras que Erc supone una historiografía estructuraß33, de forma que un cambio en el enfoque metodológico genera un cambio en la unidad de análisis de la reconstrucción histórica. En Rc, el estudio de caso es una unidad autodelimitada, en cuanto los resultados del análisis histórico se aplican fundamentalmente al caso definido como objeto de análisis; mientras que en Erc, el estudio del caso individual se motiva en la búsqueda de resultados válidos para una serie de casos que conforman el dominio de análisis. Esta es una de las razones que ha llevado a Westman (1994: 88) a afirmar que en $\mathrm{Rc}$ Kuhn maneja una narrativa histórica, pero no aún una narrativa historicista.

${ }^{33}$ Kuhn parece argumentar en la dirección opuesta cuando destaca el carácter típico de algunos problemas de la revolución copernicana (1957, p. 184), pero insistimos en considerar lateral este señalamiento, en cuanto sus consecuencias -a saber, la existencia de rasgos comunes a todas las revoluciones científicas- no son exploradas en el resto del texto.

Manuscrito - Rev. Int. Fil., Campinas, v. 38, n.3, pp. 25-57, set.-dez. 2015. 
De aquí que en $R c$ Kuhn está más próximo a lo que Floris Cohen (1994, pp. 21-24) ha denominado la 'revolución científica' como término específico, es decir como término que refiere a un período concreto de la historia de la ciencia, definido por cambios profundos en la organización del conocimiento, y ocurrido fundamentalmente a lo largo del siglo XVII ${ }^{34}$. Mientras que Erc se sitúa claramente en lo que Cohen denomina el uso genérico del término 'revolución', de acuerdo al que las revoluciones científicas son episodios que ocurren con cierta regularidad en diferentes disciplinas del conocimiento, y que dan cuenta del carácter global y abrupto del cambio teórico a lo largo de la historia ${ }^{35}$.

Estas diferencias en el alcance del análisis, por un lado dejan a $R c \sin u n$ objeto tan definido como los paradigmas -y sin el aparato filosófico que ello conlleva-, y por otro lado dan como resultado un tipo de trabajo histórico radicalmente distinto del que ofrecerá Erc. En 1957 Kuhn es ante todo un historiador de la revolución copernicana, interesado en la singularidad de ese proceso histórico y su impacto en la historia de la astronomía. Mientras que en 1962 es un historiador de la ciencia con ambiciosas pretensiones filosóficas, que busca en la revolución copernicana -lo mismo que en la revolución de la química, la óptica u otros campos- indicios de los rasgos fundamentales de toda revolución científica.

\section{Conclusión}

Se ha tendido a considerar la relación entre $R c$ de 1957 y Erc de 1962, bajo lo que hemos llamado una perspectiva continuista del pensamiento de Kuhn, para la que ambas obras dan cuenta de una misma imagen de la ciencia, que Kuhn forja sin rupturas entre las décadas de 1950 y 1960. En la discusión

\footnotetext{
${ }^{34}$ Este es el sentido que el término tiene, por ej. en la obra de A. Rupert Hall, La revolución cientifica de 1954, citada sistemáticamente por Kuhn en el texto de 1957, y sólo una vez en Erc.

35 Cohen señala también que a partir de Erc los dos sentidos se vuelven prácticamente indistinguibles, y que finalmente la idea de una revolución científica única se disuelve en el concepto genérico de las revoluciones científicas. Cabe recordar que un buen tiempo antes, Stephan Amsterdamsky (1975), había objetado a Kuhn el no distinguir entre las revoluciones científicas globales y aquellas localizadas en el interior de una disciplina.
} 
precedente hemos tratado de mostrar que esta visión del desarrollo del pensamiento kuhniano es errónea, y que la manera más adecuada de entender la relación entre sus primeras dos obras es bajo la idea de que entre ambas hay fundamentalmente una relación de discontinuidad.

Si bien las dos primeras obras de Kuhn presentan algunos supuestos historiográficos comunes, registran también diferencias de enfoque lo suficientemente agudas para no considerarlas expresión de una única visión de la ciencia, y para afirmar que cada una parte de un conjunto de presupuestos historiográficos incompatibles entre sí. Esto no conduce a que el contenido total de $R c$ y Erc sean incompatibles punto por punto, sino más bien implica que las premisas historiográficas de ambas obras -mayormente implícitas en la primera, y más bien explícitas en la segunda-, dan cuenta de formas incompatibles de comprender la ciencia, que no pueden ser integradas en una visión de conjunto.

El tenor de estas divergencias permite pensar que el viraje de Kuhn hacia el marco conceptual de Erc, sólo se termina de producir a finales de la década de los 50's, cuando su enfoque se despeja de algunos rudimentos historiográficos que operan con fuerza en Rc. En adición a esto, la obra de 1962 presenta un enfoque definidamente centrado en los paradigmas, las comunidades científicas y su dinámica interna de funcionamiento, en el que ya no cabe lugar para la consideración amplia de los factores sociales y extracientíficos, que sí tenían peso explicativo en 1957. Por otra parte, la reconstrucción de la revolución copernicana que Kuhn hace en 1957 no deja lugar para conceptos como paradigma, conversión, o inconmensurabilidad, en cuanto su obra de 1957 no presenta aún ni las implicancias ni la ambición filosófica que irrumpen en 1962. Esto involucra entre otras cosas un cambio en el tipo de proyecto historiográfico presente en cada obra, en cuanto $R c$ se centra en la revolución copernicana como objeto de estudio definido por derecho propio, mientras que Erc apunta a la detección de invariantes estructurales del conjunto de las revoluciones científicas; esto es lo que hemos llamado historiografía casuística e historiografía estructural, respectivamente.

Entendemos que la consideración de estas reorientaciones es relevante para desmontar la imagen -parcialmente fomentada por el mismo Kuhn- de un proyecto filosófico que comienza a gestarse a finales de la década de los 40's, y que se desenvuelve armónica y acumulativamente hasta llegar a Erc. Esta consideración continuista del pensamiento de Kuhn, sólo puede sostener se si se pasan por alto las divergencias y se amplifican las continuidades de su 
pensamiento, premisa metodológica que a nuestro entender oculta más de lo que revela. Esto en cuanto impide dar cuenta de una serie de rupturas que experimenta el pensamiento de Kuhn luego de $\mathrm{R} c$ y previo a Erc, cuya consideración permite entender que el enorme impacto de la obra mayor de Kuhn se debe -entre otras cosas- a que introduce herramientas conceptuales que no estaban presentes -en algunos casos siquiera en germen- en su trabajo temprano.

\section{Referencias}

Amsterdamski, S. Between Experience and Metaphysics: Philosophical Problems of the Evolution of Science. BSPS XXXV. Dordrecht/Boston: D. Reidel Pub. Co., 1975.

BIRD, A. Thomas Kubn. Madrid: Tecnos, 2000.

."Kuhn and the Historiography of Science". In DEVLIN, W.; BOKULICH, A. (eds.) (2015), pp. 23-38.

Bravo, E. "Thomas S. Kuhn, La revolución copernicana". Crítica. Revista Hispanoamericana de filosofía, 11, 31, pp. 140-147, 1979.

CoHen, F. The Scientific Revolution. An Historiographical Inquiry. Chicago: The University of Chicago Press, 1994.

ConAnt, J. B. On Understanding Science. New Haven: Yale University Press, 1947.

DEVLIN, W.; BOKULICH, A. (eds.), Kubn's Structure of Scientific Revolutions - 50 Years On, BSPS, 311. Dordrecht: Springer, 2015.

ESTRADA, R. "Dos historias de la ciencia: Kuhn y Koyrè”. En PÉREZ Ransanz, A. R. (ed.) (2005), pp. 137-160.

FEINGOLD, M. (ed.), Jesuit Science and the Republic of Letters. Massachusetts: MIT Press, 2003.

FIscher, K. Galileo Galilei. Barcelona: Herder, 1986. [1º ed. 1980]

Grandy, R. “Kuhn’s World Changes”. In NiCKLES, T. (ed.) (2003), pp. 246260.

Manuscrito - Rev. Int. Fil., Campinas, v. 38, n.3, pp. 25-57, set.-dez. 2015. 
GENTILE, N. La tesis de la inconmensurabilidad. A 50 años de La estructura de las revoluciones cientificas. Buenos Aires: EUdeBA, 2013.

Guillaumin, G. Historia y estructura de La estructura. Origen del pensamiento histórico de Thomas Kubn. México: Universidad Autónoma Metropolitana, 2012.

HeILBRon, J. L. “Thomas Samuel Kuhn”. Isis, 89, 3, pp. 505-515, 1998.

HeILBRON, J. L.; KuHN, T. S. "The genesis of the Bohr atom". Historical Studies in the Physical Sciences, 1, pp. 211-290, 1969.

Hoyningen-Huene, P. Reconstructing Scientific Revolutions. Chicago: The University of Chicago Press, 1993.

- "Philosophical Elements in Thomas Kuhn's Historiography of Science". Theoria, 75, pp. 281-292, 2012.

KuHN, T. The Copernican Revolution. Cambridge-Mass.: Harvard University Press, 1995 -eighteenth printing-. [1º ed. 1957]

- La revolución copernicana. Barcelona: Ariel, 1996. [1º ed. 1957]

. The Structure of Scientific Revolutions. Chicago: The University of Chicago Press, $19702^{\text {nd }}$ edition (1 ${ }^{\text {st }}$ edition 1962).

- The Structure of Scientific Revolutions. 1961 Draft. Available: https://unihannover.academia.edu/PaulHoyningenHuene

—. La estructura de las revoluciones científicas. Introducción de Ian Hacking. Traducción e introducción de Carlos Solís. México: FCE, 2013. [1 ed. 1962]

—_ "La función de la medición en la física moderna", 1961. En La tensión esencial, pp. 202-247.

—. "La estructura histórica del descubrimiento científico", 1962. En La tensión esencial, pp. 189-201.

_- "La historia de la ciencia", 1968. En La tensión esencial, pp. 129-150.

_ . "Las relaciones entre la historia y la historia de la ciencia", 1971. En La tensión esencial, pp. 151-185.

- The essential tension. Chicago: The University of Chicago Press, 1977.

Manuscrito - Rev. Int. Fil., Campinas, v. 38, n.3, pp. 25-57, set.-dez. 2015. 
—. La tensión esencial. México: FCE, 1982. [1º ed. 1977]

. "Una conversación con Thomas Kuhn. Entrevista con Arístides Baltas, Kostas Gavroglu y VAssiliki Kindi”, 1995. En El camino desde La Estructura (2002), pp. 301-373.

-. En El camino desde La Estructura. Barcelona: Paidós, 2002. [1 ${ }^{\circ}$ ed. 2000]

MARCuM, J. Thomas Kuhn's Revolution: An Historical Philosophy Of Science. New York: Continuum, 2005.

MAYORAL, J. "Las conferencias Lowell de Kuhn: un estudio crítico". Theoria, 78, pp. 459-476, 2013.

MelognO, P.; ACHe, S. "De los esquemas conceptuales a los paradigmas. Evolución del pensamiento de Thomas Kuhn, 1957-1962”. En Acta Scientiarum. Human and Social Sciences, 37, 2, 2015 (en prensa).

-.; PELEGRIN, D. "Historia e inconmensurabilidad: en torno a la práctica filosófica de Thomas Kuhn”. En MELOGNO, P.; PELEGRIN, D. (comps.) (2015), pp. 89-102.

- (comps.), Realismo, verdad y significado. Montevideo: Índice Grupo Editorial/ Facultad de Información y Comunicación-Universidad de la República, 2015.

NiCKLES, T. (ed.), Thomas Kuhn. Cambridge-UK: Cambridge University Press, 2003.

PARDO, C. La formación intelectual de Thomas Kubn, Una aproximación biográfica a la teoría del desarrollo cientifico. Pamplona: Eunsa, 2001.

Pérez Ransanz, A. R. Kubn y el cambio científico. México: FCE, 1999.

- (ed.), Perspectivas y horizontes de la filosofía de la ciencia a la vuelta del tercer milenio. Vol. II. México: UNAM, México, 2005.

Pinto De Oliveira, J.C. "Kuhn and the genesis of the "new historiography of science". Studies in History and Philosophy of Science, 43 (A), 1, pp. 115-121, 2012.

RUPERT HALL, E. La revolución científica. Barcelona: Crítica, 1985. [1º ed. 1954] 
SwerdLow, N. “An Essay on Thomas Kuhn's First Scientific Revolution, The Copernican Revolution". Proceedings of the American Philosophical Society, 148, 1, pp. 64-120, 2004.

Westman, R. “Two Cultures or One?: A Second Look at Kuhn's The Copernican Revolution”. Isis, 85, 1, pp. 79-115, 1994.

Manuscrito - Rev. Int. Fil., Campinas, v. 38, n.3, pp. 25-57, set.-dez. 2015. 
